\title{
Multiplex Probe Parameter
}

National Cancer Institute

\section{Source}

National Cancer Institute. Multiplex Probe Parameter. NCI Thesaurus. Code C116118.

A description of the number of unique molecular probes used in each assay well or tube. 